\title{
SIR model of epidemic spread with accumulated exposure
}

\author{
B. Dybiec ${ }^{\mathrm{a}}$
}

M. Smoluchowski Institute of Physics, and Mark Kac Center for Complex Systems Research, Jagellonian University, ul. Reymonta 4, 30-059 Kraków, Poland

and

Center for Models of Life, Niels Bohr Institute, Blegdamsvej 17, 2100 Copenhagen Ø, Denmark

Received 27 December 2007 / Received in final form 27 October 2008

Published online 4 December 2008 - (c) EDP Sciences, Società Italiana di Fisica, Springer-Verlag 2008

\begin{abstract}
We study an extended and modified SIR model of epidemic spread in which susceptible agents during interactions with infectious neighbors are exposed to the disease and can consequently become infectious. The studied model is extended to include heterogeneity of interactions which is modelled assuming random character of the dose accumulated by susceptible agents in every interaction with infectious neighbors. When the accumulated exposure is larger than the individual's resistance, an agent becomes infectious and consequently introduces a new source of an epidemic which is capable of passing the disease further. We study statistical properties characterizing the course of an epidemic. The examination of the modified SIR model reveals a possible 'resonant activation'-like behavior of the system in the duration of the epidemic outbreak and a possible bistable behavior of the model with accumulated exposure. Furthermore, the linear scaling of the duration of the epidemic with the system size for a wide range of the model parameters is recorded.
\end{abstract}

PACS. 87.23.Cc Population dynamics and ecological pattern formation - 82.20.Wt Computational modeling; simulation - 05.50.+q Lattice theory and statistics (Ising, Potts, etc.) - 05.10.Ln Monte Carlo methods

\section{Introduction}

Computer simulations of cellular automata [1,2] are considered as one of possible methodologies for investigation of epidemiological models [3,4]. The cellular automata are especially suitable for that purpose because it is usually assumed that agents building the society can be in a discrete number of states. A basic group of epidemiological models originates from the SIR (Susceptible-InfectiousRecovered) model [5] which is, on the one hand, often modified by introduction of additional states of the individual $[4,6]$. On the other hand, the interplay between agents and the assumed system topology is another field of possible modification of the considered models [4,7].

It is frequently assumed that transitions of agents between possible states take place with a constant probability $[8,9]$. However, some transitions (like infection) are neighborhood dependent and require that a given agent is in contact with at least one infectious agent [10]. If a susceptible agent is in contact with a larger number of infectious individuals, it can become infectious with a higher probability. This originates not from a larger probability of infection in a single interaction but from a larger number of trials. A mathematical explanation of this fact is provided by the Bernoulli scheme. Therefore, connectivity of a network introduces spatial inhomogeneity. Nevertheless,

\footnotetext{
a e-mail: bartek@th.if.uj.edu.pl
}

it is often assumed that all individuals are equally resistant, i.e. they are homogeneous. Consequently, homogeneity and heterogeneity mix in the same model.

Here we propose a possible method which further extends the standard SIR model. We assume that space is homogeneous, i.e. all agents are placed onto a regular square lattice. However, we modify the character of interactions between individuals by modifying the disease transmission process. We assume that during interactions with an infectious individual every susceptible individual is exposed to a random dose of infection which is accumulated, which in turn is equivalent to the accumulation of the probability to become infected. The interplay between the accumulated exposure and the agent's resistance determines whether the susceptible agent becomes infectious or not. The stochastic character of exposure accounts for inhomogeneity of contacts between connected individuals. In other words, depending on the situation, the link existing in the system can be used for transmission of germs, however, the amount of the transmitted pathogen is not constant for every interaction.

The main goal of current investigations is a statistical analysis of the the SIR model of the epidemic spread with accumulated exposure. In particular, we analyze individuals' residence times in possible states, the duration of the epidemic and its severity measured by the fraction of individuals affected by an epidemic outbreak. The paper is organized in the following way: in the next section 
(Model) we present the model in question. The findings regarding statistical properties of the epidemic ruled by the suggested model are included and discussed in the results section. Finally, the paper is closed with some concluding remarks (Summary and conclusions).

\section{Model}

The suggested extended version of the SIR model has two basic, closely connected, elements: (1) topology and (2) individuals. The present topology determines possible interactions between individuals, i.e. it contains information about all possible connections joining individuals building the system. Individuals themselves are placed onto the chosen topology and can be in a discrete number of states. We also assume that all nodes in a network are occupied.

We choose the simplest topology possible, i.e. a regular, square lattice topology with periodic boundary conditions. Each individual present in the system can interact with four of its nearest neighbors (von Neumann neighborhood). Nevertheless, already this simple topology together with the considered shape of interactions generates the rich behavior of the studied model.

For the epidemiological part of the model we choose a SIR model [5]. The initial state is a mixture of the majority of susceptible individuals, with the addition of 10 infectious individuals I. A susceptible individual, $\mathbf{S}$, can be infected after accumulating exposure greater than its infection threshold (resistance), IT, [11]. The exposure originates from contact with any infectious individual in its neighborhood. Upon successful infection an agent moves to the infectious class, $\mathbf{I}$. An infectious individual can subsequently move to the recovered class, $\mathbf{R}$, (with probability $r$ of a spontaneous recovery). The transitions and their conditions are summarized in the following graph

$$
\mathbf{S} \stackrel{\exp >I T}{\longrightarrow} \mathbf{I} \stackrel{r}{\longrightarrow} \mathbf{R}
$$

The individual's resistance is constant over time and space, i.e. all individuals in the system are characterized by the same infection threshold. However, the relative resistance of individuals, due to the accumulation of the exposure to disease, can vary over time. Exposure doses are assumed to be nonnegative and distributed according to symmetric stable distributions restricted to nonnegative values only. More precisely, if a generated exposure dose is negative, the absolute value of the exposure dose is taken. Symmetric stable distributions with the zero mean are characterized by their Fourier-transforms (characteristic functions of the distributions) being $[12,13]$

$$
\phi(k)=\exp \left[-\sigma^{\alpha}|k|^{\alpha}\right] .
$$

Here, the most important parameter $\alpha, 0<\alpha \leqslant 2$, is the stability index of the distribution, which describes its asymptotic power-law behavior, i.e. for $\alpha<2$ with large $x P(x) \propto|x|^{-(1+\alpha)}$. In general, stable random variables correspond to the four-parameter family of random variables $[12,13]$. Here, we use only a limited subset of stable variables, i.e. symmetric ones with the zero mean corresponding to $1 \leqslant \alpha \leqslant 2$. Well-known examples of stable distributions include the Cauchy distribution $(\alpha=1)$ and the Gaussian distribution $(\alpha=2)$. The random numbers distributed according to characteristic functions given by equation (2) can be generated using Janicki-Weron algorithm [13-15]. In the simulations we assume $\sigma=1$.

During the simulation, the system was updated synchronously as long as there were any infectious individuals left. To guarantee a finite length of simulation, a nonzero probability of spontaneous recovery, $r$, was assumed. The duration of the epidemic, which is measured from the occurrence of first infectious individuals till the recovery of the last infectious individual, introduces the maximal value of the residence time in possible states $(\mathbf{S}, \mathbf{I}, \mathbf{R})$, which is smaller than the duration of the epidemic outbreak. The properties of the system were examined by running the simulation $5 \times 10^{3}$ times and collecting values of residence times for each run. The basic size of the system is $100 \times 100$. However, results of simulations with other systems sizes are in the qualitative agreement with the results for the basic system size $100 \times 100$.

\section{Results}

The duration of the epidemic and residence times of individuals in possible states are not independent. There exists the following connection between duration of the epidemic in $i$ th realization, $\tau_{i}$, and residence time in $\mathbf{S}, \mathbf{I}, \mathbf{R}$ classes

$$
\tau_{i}=\max _{j}\left[\tau_{i}^{j}\right]=\max _{j}\left[\left(\tau_{S}+\tau_{I}\right)_{i}^{j}\right]
$$

$\left(\tau_{\ldots}\right)_{i}^{j}$ denote the residence time in a given state $(\mathbf{S}, \mathbf{I}, \mathbf{R})$ of the individual $j$ during the $i$ th realization of the simulation. Equation (3) emerges as a consequence of the fact that the duration of the epidemic outbreak is determined by the maximal length of the epidemic experienced by a single individual and the fact that the epidemic outbreak ends when the last infectious individual moves to the recovered class. Equation (3) implies that $\left(\tau_{S}+\tau_{I}+\tau_{R}\right)_{i}^{j} \leqslant \tau_{i}$ for every $j$. In the performed simulations $i \in\left\{1,2, \ldots, 5 \times 10^{3}\right\}$ and $j \in\{1,2, \ldots, 100 \times 100\}$. Properties of duration of the epidemic are studied by use of a $\tau_{i}$ s sample, while the average residence times are inspected from a samples of collected $\left(\tau_{\ldots}\right)_{i}^{j}$. More precisely, $\langle\tau\rangle=\left\langle\tau_{i}\right\rangle,\left\langle\tau_{S}\right\rangle=\left\langle\left(\tau_{S}\right)_{i}^{j}\right\rangle,\left\langle\tau_{I}\right\rangle=\left\langle\left(\tau_{I}\right)_{i}^{j}\right\rangle,\left\langle\tau_{R}\right\rangle=\left\langle\left(\tau_{R}\right)_{i}^{j}\right\rangle$, i.e. $\langle\tau\rangle$ is averaged over $5 \times 10^{3}$ elements and $\left\langle\tau_{\ldots}.\right\rangle$ are averaged over $5 \times 10^{3} \times 100^{2}$ elements.

The definition of the duration of the epidemic (3) is of the extreme statistics type. On the one hand, it can be significantly extended by extreme events. For example, by individuals spending a very long time in the infectious state or becoming infectious very late. On the other hand, such a definition assures that indeed all cases of the epidemic have been successfully eradicated. This issue is very important in the case of world wide epidemics such as SARS [16] or avian influenza [17] when it was necessary 

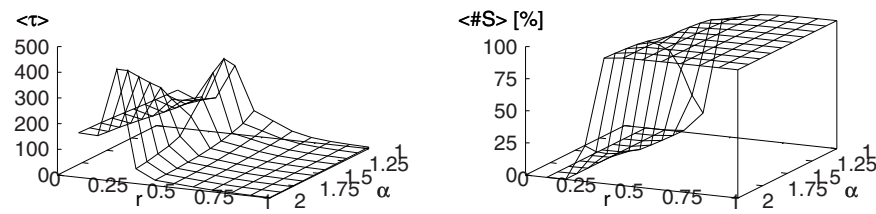

Fig. 1. Average duration of the epidemic, $\langle\tau\rangle$, (left panel) and average number of individuals in the susceptible state, $\langle \# S\rangle$, at the end of the epidemic as a fraction of the total number of individuals (right panel). Initially, there were $I_{0}=10$ infectious individuals in the system. The infection threshold $I T=3$.

to completely eradicate all cases in order to stop the epidemic. However, even this approach does not provide full security, because epidemics already considered as eradicated can reappear. Alternative definitions of the duration of the epidemic can be based on the fraction of individuals in the infectious state. For example, an epidemic can be considered as finished, when number of infected individuals drops below a certain threshold or when all known cases are perfectly isolated. If the threshold is equal to zero, this kind of definition is equivalent to the one considered here. Higher values of the threshold, as less secure and potentially dangerous, are not considered here.

Figure 1 presents the average duration of the epidemic, $\langle\tau\rangle$, (right panel) and the average number of susceptible individuals at the end of the epidemic, $\langle \# S\rangle$, (left panel) as a function of the recovery probability, $r$, and the exponent, $\alpha$, characterizing the distribution of exposure doses. All individuals in the system are characterized by the same resistance level which in Figure 1 is adjusted to $I T=3$. In the studied system there is a bistable behavior, i.e. there is a sharp transition between the presence or lack of the epidemic outbreak, see right panel of Figure 1. A short duration of the epidemic corresponds to the situation when the only observed process is the recovery of initially infectious individuals. In a situation when there is no the epidemic outbreak the duration of a simulation is determined by the recovery of initially infectious individuals because the resistance of individuals is high enough to prevent the outbreak.

Figures 2-4 show results presenting the influence of the recovery probability, $r$, the infection threshold, $I T$, the exponent characterizing distributions of exposure doses, $\alpha$, and the system size on the character of the observed epidemic outbreaks. The depicted curves are extracted from surfaces of the type presented in Figure 1. Every figure presents: the average duration of the epidemic, $\langle\tau\rangle$, (top left panel), the average residence time in the susceptible state, $\left\langle\tau_{S}\right\rangle$, (top right panel), the average number of individuals in the susceptible state, $\langle \# S\rangle$, at the end of the epidemic as a fraction of the total number of individuals (bottom left panel) and the average residence time in the recovered state, $\left\langle\tau_{R}\right\rangle$, (bottom right panel). Figure 2 presents results for $\alpha=1.5$ with various infection thresholds $I T=\{0.75,1.0,1.25,3.0,7.0\}$. Figure 3 demonstrates the role of the stability index $\alpha$ on the course of the epidemic by comparison of results for $\alpha=\{1.1,1.5,1.9\}$ with $I T=1.25$. Figure 4 shows results for different system sizes $50 \times 50,100 \times 100$ and $200 \times 200$ with $\alpha=1.5$ and $I T=3$.
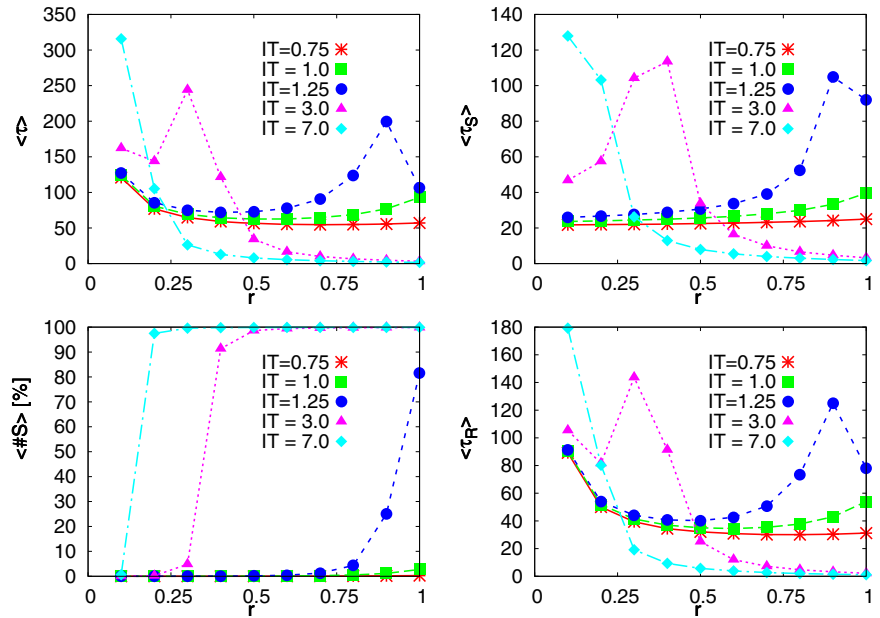

Fig. 2. (Color online) Average duration of the epidemic, $\langle\tau\rangle$, (top left panel), the average residence time in the susceptible state, $\left\langle\tau_{S}\right\rangle$, (top right panel), the average number of individuals in the susceptible state, $\langle \# S\rangle$, at the end of the epidemic as a fraction of the total number of individuals (bottom left panel) and the average residence time in the recovered state, $\left\langle\tau_{R}\right\rangle$, (bottom right panel). Initially, there were $I_{0}=10$ infectious individuals in the system. Various curves correspond to various levels of the infection threshold $I T=\{0.75,1.0,1.25,3.0,7.0\}$. The size of the system is $100 \times 100$. The stability index characterizing the power-law behavior of the probability density is $\alpha=1.5$.
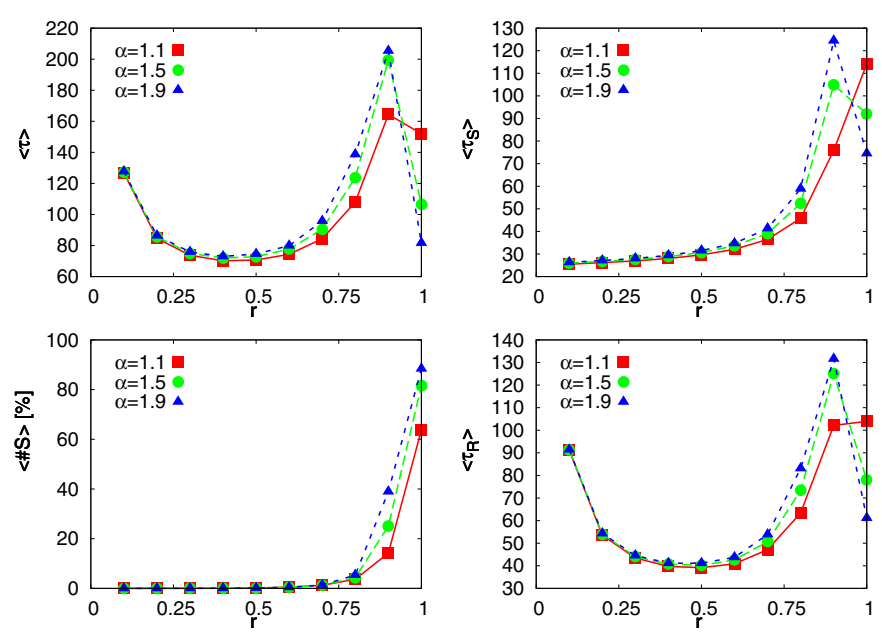

Fig. 3. (Color online) The same as in Figure 2 for various $\alpha=$ $\{1.1,1.5,1.9\}$. The system size is $100 \times 100$. Infection threshold $I T=1.25$.

Finally, Figure 5 further explores the dependence of the model on the system size.

In the final state of an epidemic, there are no infectious individuals in the system because all of them have moved to the recovered class. Consequently, in the system there can be only susceptible or recovered individuals. Furthermore, due to a constant size of the population, the number of recovered individuals at the end of the epidemic is related to the number of susceptible individuals. From the bottom left panels of Figures 2-4 it is possible to determine the cases when the epidemic outbreak does or does 

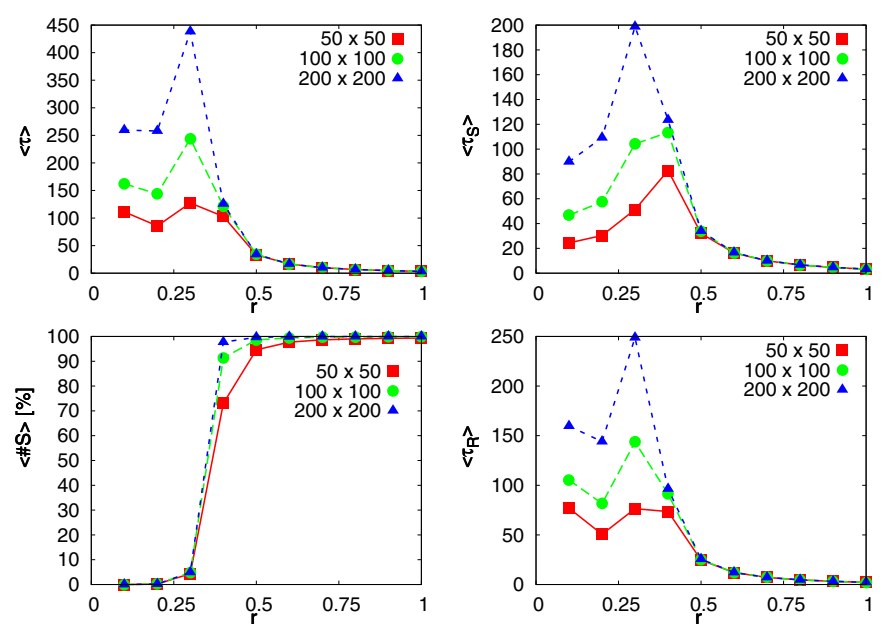

Fig. 4. (Color online) The same as in Figure 2 for various system sizes. The stability index characterizing the power-law behavior of the probability density $\alpha=1.5$. Infection threshold $I T=3$.

not take place. For $I T \geqslant 2$, in the studied model, pronounced bistable behavior is observed. For small values of the recovery probability there is an epidemic outbreak, while for large values of recovery probability there is no the epidemic outbreak. The larger the epidemic threshold, the smaller the recovery probability at which the epidemic outbreak takes place.

The behavior of the model is determined by an interplay between two processes. The first process is the accumulation of exposure doses. This process is determined by the power-law exponent, $\alpha$, and the resistance of individuals, IT. The biological motivation for this process is discussed in Summary and conclusions section. The next process is recovery of infectious individuals which is characterized by the recovery probability, $r$. The recovery probability determines for how long an infectious individual can infect its neighbors. Increasing the resistance level increases the time which is required to accumulate exposure larger than the infection threshold. Consequently, for a larger infection threshold it is harder to infect susceptible individuals.

For a small infection threshold, e.g. $I T \leqslant 1$, for all values of the recovery probability, $r$, practically all individuals go through the disease and consequently a severe epidemic outbreak is observed. For $I T<0.75$, the epidemic duration is a monotonic function of the recovery probability, $r$. A large average duration of the epidemic corresponds to a small value of the recovery probability which is associated with a long recovery time. The situation changes when the resistance of individuals increases. In such a case a larger number of interactions is necessary to infect susceptible individuals. Consequently, for a large recovery probability, $r$, a longer time is necessary. This is caused by the fact that the recovery process is relatively fast and infectious neighbors move to the recovered class which in turn leads to a situation where exposure to infection can be acquired from a smaller number of neighbors. This situation is manifested by the non-monotonic
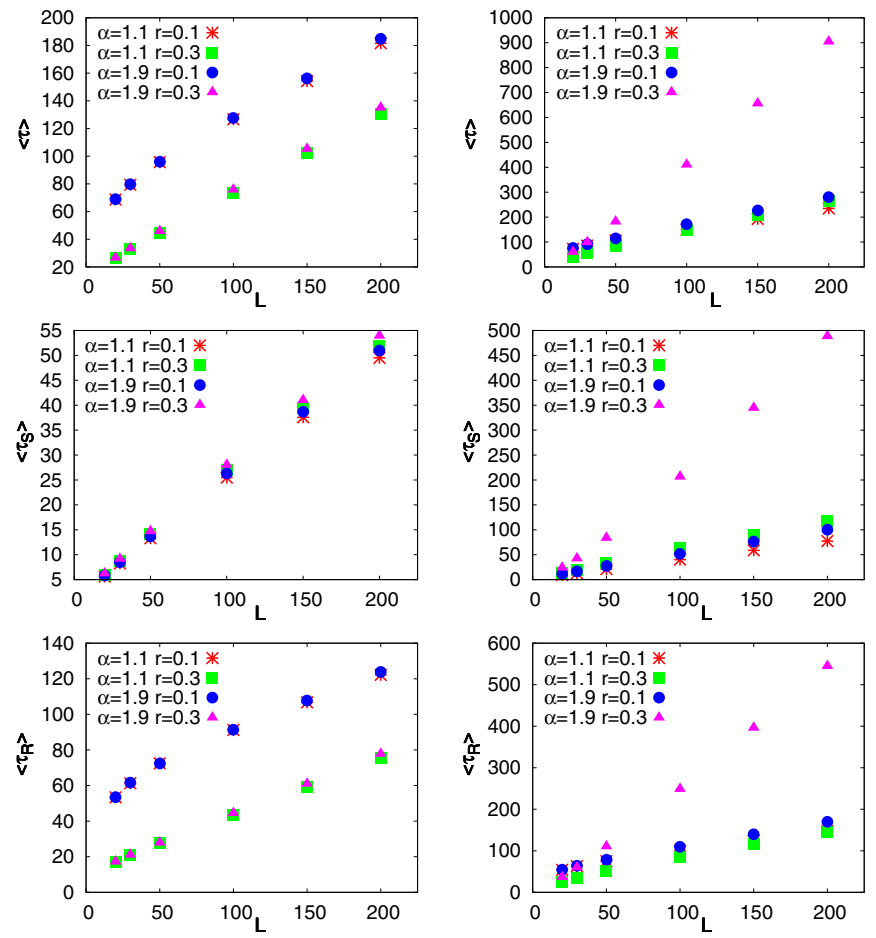

Fig. 5. (Color online) The average duration of the epidemic $\langle\tau\rangle$ (top panel), the average residence time in the susceptible state $\left\langle\tau_{S}\right\rangle$ (middle panel) and the average residence time in the recovered state $\left\langle\tau_{R}\right\rangle$ (bottom panel) for $\alpha=\{1.1,1.9\}$ and $r=\{0.1,0.3\}$. Infection threshold $I T=1.25$ (left panel) and $I T=3$ (right panel). Initially, there were $I_{0}=10$ infectious individuals in the system.

dependence of the average duration of the epidemic as the function of the recovery probability, $r$, see left top panels of Figures 2-4. With a further increase of the infection threshold, the number of individuals going through the disease decreases. Finally, for large values of resistance, the recovery process is fast enough to prevent the epidemic outbreak, see bottom left panels in Figures 2-4. This also explains why with an increasing infection threshold the maxima of the epidemic duration move to smaller values of the recovery probability, $r$.

On the one hand, large values of the duration of the epidemic for small values of the recovery probability, $r$, are further increased by the recovery process, i.e. by the time which individuals spend in the infectious state. On the other hand, the maxima of the epidemic length observed for large values of the recovery probability, $r$, are produced by a slow infection process. This fact is further corroborated by inspection of the average residence time in the susceptible state, $\left\langle\tau_{S}\right\rangle$, see top right panels in Figures $2-4$. The large value of the average residence time in the susceptible state in situations when all individuals go through the disease indicates that the infection process is slow. Therefore, on average susceptible individuals become infectious lately at the moment which is relatively close to the duration of the epidemic.

The average residence time in the recovered class $\left\langle\tau_{R}\right\rangle$ is the average value of residence times $\left(\tau_{R}\right)_{i}^{j}$ such that $\left(\tau_{S}+\tau_{I}+\tau_{R}\right)_{i}^{j} \leqslant \tau_{i}$. In other words, the average residence 
time in the recovered state is the average value of time intervals between the recovery of individuals and the end of the epidemic. It characterizes the average time which is spent in the recovered class by recovered individuals before the final end of the epidemic. The qualitative behavior of the $\left\langle\tau_{R}\right\rangle$ is of the same type as behavior of $\tau_{i}$, compare top left vs. bottom right panels in Figures 2-4.

The duration of the epidemic, as well as the average residence time in the recovered state, can be a nonmonotonic function of the recovery probability, see Figure 3. These effects can be explained by the interplay of the infection process, the recovery process and the epidemic threshold IT. In Figure 3, for a small recovery probability, $r$, all individuals become infectious quickly and all of them go through the disease. The duration of the epidemic is extended by the long time which individuals spend in the infectious state due to the slow recovery rate. With increasing recovery probability the infection process is fast enough for all individuals to become infectious. However, the recovery process is fast enough not to extend the duration of the epidemic by the long waiting time for the recovery. Further increase of the recovery probability decreases the speed of the epidemic spread, because infected individuals quickly move to the recovered state. Consequently, at each time there is a significantly smaller number of infectious individuals than for a low recovery probability, which contributes to further the epidemic spread. Therefore, the duration of the epidemic is further increased. The non-monotonic dependence of the average residence time in the recovered state is explained analogously. For a small recovery probability, recovered individuals have to wait for an individual which recovered last, while for a large recovery probability, recovered individuals wait for an individual which becomes infected as the last one.

Possible non-monotonic dependence of the average residence time in the susceptible state is explained by the interplay of the infection process, recovery process and the infection threshold, as well. In order to observe the epidemic outbreak which spans the whole system, the infection process has to be fast enough to survive outflow of recovering individuals. The decreasing number of simultaneously infected individuals decreases the rate of the epidemic spread. This corresponds to the increasing part of the $\left\langle\tau_{S}\right\rangle$ curves. A possible further decrease of the mean residence time in the susceptible state corresponds to the situations when the recovery process is fast enough and the resistance large enough to prevent epidemic outbreaks. In such a situation, the main process is the recovery process and consequently, the duration of the epidemic is short and, in turn, leads to a small value of the residence time $\tau_{S}$ and the low epidemic severity. Additionally, the above mentioned effects are further confirmed by the monotonic dependence of the average number of interactions which need to take place in order to infect a given susceptible individual characterized by the residence $I T$ as a function of the stability exponent $\alpha$.

The behavior of the residence time in the infectious state is the straightforward consequence of the purely probabilistic character of transitions from the infectious, I, to the recovered, $\mathbf{R}$, class. These transitions are not coupled to the state of the neighborhood. The residence time distribution in the infectious state is $P\left(\tau_{I}=i\right)=$ $r(1-r)^{i-1}$. The distribution is fully determined by the recovery probability $r$. Consequently, the average residence time $\left\langle\tau_{I}\right\rangle$ is equal to one over recovery probability $r$, namely $1 / r$.

For small values of the resistance level, it is easy to overcome the infection threshold and consequently the considered model is not sensitive to the choice of the stability index, $\alpha$. The situation slightly changes for larger values of the infection threshold, when it is easier to become infectious for smaller values of $\alpha$ for which larger exposure doses are more likely. However, these changes are minimal, see Figure 3.

With the increasing system size, the duration of the epidemic increases. The bistable behavior of the model is more apparent. The transition between the situation when there is an epidemic outbreak and there is no outbreak is more rapid. However, for each considered system size, results are in the qualitative agreement, see Figure 4.

Figure 5 presents: the average duration of the epidemic $\langle\tau\rangle$ (top panel), the average residence time in the susceptible state $\left\langle\tau_{S}\right\rangle$ (middle panel) and the average residence time in the recovered state $\left\langle\tau_{R}\right\rangle$ (bottom panel) for the resistance level $I T=1.25$ (left panel) and $I T=3$ (right panel) with $\alpha=\{1.1,1.9\}$ and $r=\{0.1,0.3\}$. Exact values of the parameters are indicated in the figures' legends. For the chosen set of parameters these times depend linearly on the system size. In general, there exists a wide range of parameters for which linear scaling is visible. For $I T \leqslant 1.25$ linear scaling is visible for all values of the recovery probability, $r$, and the stability index, $\alpha$. With the increasing resistance of individuals the maximal value of the recovery probability, $r$, below which there is the linear scaling, decreases. For example, for $I T=3$ the linear dependence is visible for $r \leqslant 0.3$ while for $I T=7$ for $r \leqslant 0.1$. For small values of $\alpha$ the linear dependence is more persistent, i.e. for a given value of IT the threshold value for small $\alpha$ can be a little bit higher than for a larger $\alpha$. The linear dependence on the system size visible in Figure 5 is the manifestation of the fast infection process which leads to the situations when all or a significant ratio of individuals become infected, see Figures 2-4. The slowing down of the infection process caused either by the fast recovery process or high level of individuals' resistance leads to deviation from the linear scaling. In such cases, the dependence on the system size becomes irregular (results not shown).

\section{Summary and conclusions}

The SIR model provides a basic framework for the investigation of the epidemic spread. Here, we extend the SIR model by modifying the conditions of the infection. In the studied model, a susceptible agent becomes infectious after accumulating exposure larger than its resistance level. 
The size of the exposure dose varies from interaction to interaction. This variability accounts for a spatial dynamical inhomogeneity of the system. Furthermore, the variability of the exposure dose amplifies the stochastic character of interactions between individuals.

The studied model is derived from the real life observation that it is possible to become infected after a single interaction with a very infectious individual or after a series of interactions with less infectious individuals. The mentioned observation suggests that at least to some extent the infectious agent is accumulated during interactions between infectious and susceptible individuals. The studied model can be interpreted as the model of the infection transmission in an inhomogeneous population with fluctuations in infectiveness. In such an interpretation, interactions between individuals lead to possible changes in the probability of becoming infected. Every interaction between infectious and susceptible individual increases the probability that the susceptible individual becomes infected. Therefore, the interactions can be considered as the accumulation of the probability to get infected. The changes in the probability to get infected result from a highly intermittent process which is approximated by the considered process of the accumulation of pathogen. Here, it is assumed that these changes are generated from the power-law distribution (with a finite mean). This corresponds to the well-known observation of social systems in which the topology of interactions among individuals often has a scale-free character, i.e. the number of interactions or friends belongs to a power-law distribution. The scale-free character of the underlying system topology can produce power-law distributions of transmitted quantities of pathogen which are assumed here.

The studied model describes transitions from the initial to the final state. The initial state is the susceptible state with a small addition of infectious individuals. The final state, due to a nonzero recovery probability, is absorbing. In the absorbing state all individuals are in the susceptible or recovered state. By altering values of simulation parameters it is possible to change the character of the transition from the initial to the final state. For a small value of the infection threshold, all individuals regardless of the recovery probability go through the disease. For moderate values of the infection threshold, the characteristic time associated with such a transition can be a non-monotonic function of the recovery probability. This kind of behavior is very similar to the effect of the resonant activation $[18,19]$. For large values of the infection threshold, only a part of the system goes through the infectious state because the recovery process is fast enough to prevent accumulation of exposure larger than the resistance of individuals. Finally, for very large resistance level, the only observed process is the recovery of infectious individuals.

The studied model belongs to a general epidemic process [10] similar to one considered in [20]. However, there are differences between both models. The properties of percolation on heterogeneous networks [20] are determined by the weakest links due to the fact that they are hardest to pass the epidemic through. Consequently, the weakest links introduce a kind of firewall which is able to prevent susceptible individuals from becoming infectious by creating a kind of protective ring. The model of accumulated exposure also included heterogeneity, however, the heterogeneity is introduced on the level of interactions. All links in the system have the same weights but the amount of pathogen transmitted over them varies over time. Furthermore, it is assumed that initially all individuals in the system are equally resistant. In the course of time, when individuals have accumulated pathogen, their relative resistance decreases. Therefore, if an individual was not infected in previous interactions, it becomes less resistant and in turn it can be easier infected. The process of accumulation of pathogen corresponds to increasing weights assigned to links [20]. The process of accumulating the pathogen is weakened by recovery of infectious neighbors which in cooperation with infection process is responsible for observed effects.

In our opinion, the suggested extensions to the SIR model make it more realistic by incorporating the stochastic heterogeneity of interaction between agents building the system. Heterogeneity can be further extended by modification of the structure of interaction between individuals, i.e. topology. For example, instead of a regular lattice topology it is possible to consider a small world topology $[4,21]$ or a scale-free topology [22]. This kind of topology should further amplify the power-law distributions of accumulated doses of infection. Furthermore, it is also possible to include variability in the resistance level $[6,23,24]$ which can introduce further spatial heterogeneity $[25,26]$.

The research has been supported by the Foundation for Polish Science through the Foreign Postdoc Fellowship. The support by the Marie Curie TOK COCOS grant (6th EU Framework Programme under Contract No. MTKD-CT-2004-517186) is also acknowledged. Additionally, B.D. acknowledges hospitality of the Niels Bohr Institute (Copenhagen). Special thanks are directed to Kim Sneppen for fruitful and inspiring discussions.

\section{References}

1. S. Wolfram, Rev. Mod. Phys. 55, 601 (1983)

2. S. Wolfram, A New Kind of Science (Wolfram Media, Champaign, IL, 2002)

3. C. Rhodes, R. Anderson, Theor. Popul. Biol. 52, 101 (1997)

4. B. Dybiec, A. Kleczkowski, C.A. Gilligan, Phys. Rev. E 70, 066145 (2004)

5. R.M. Anderson, R.M. May, Infectious Diseases of Humans: Dynamics and Control (Oxford University Press, Oxford, 1991)

6. E. Ahmed, H.N. Agiza, Physica A 253, 347 (1998)

7. N. Boccara, K. Cheong, J. Phys. A: Math. Gen. 26, 3707 (1993)

8. B. Dybiec, A. Kleczkowski, C.A. Gilligan, A. Phys. Pol. B 36, 1509 (2005) 
9. A. Kleczkowski, B. Dybiec, C.A. Gilligan, A. Phys. Pol. B 37, 3017 (2006)

10. P. Grassberger, Math. Biosci. 63, 157 (1983)

11. S. Sadedin, B. Dybiec, G. Briscoe, Physica A 323, 715 (2003)

12. W. Feller, An introduction to Probability Theory and its Applications (John Wiley, New York, 1968)

13. A. Janicki, A. Weron, Simulation and Chaotic Behavior of $\alpha$-Stable Stochastic Processes (Marcel Dekker, New York, 1994)

14. A. Weron, R. Weron, Lecture Notes in Phys. 457, 379 (1995)

15. R. Weron, Statist. Probab. Lett. 28, 165 (1996)

16. C. Dye, N. Gay, Science 300, 1884 (2003)

17. V. Colizza, A. Barrat, M. Barthelemy, A. Valleron, A. Vespignani, PLoS Med 4, e13 (2007)
18. C.R. Doering, J.C. Gadoua, Phys. Rev. Lett. 69, 2318 (1992)

19. B. Dybiec, E. Gudowska-Nowak, Phys. Rev. E 69, 016105 (2004)

20. L.M. Sander, C.P. Warren, I.M. Sokolov, C. Simon, J. Koopman, Math. Biosci. 180, 293 (2002)

21. D.J. Watts, S.H. Strogatz, Nature 393, 440 (1998)

22. R. Albert, A.L. Barabasi, Rev. Mod. Phys. 74, 47 (2002)

23. Q. Liu, Z. Jin, Chinese Phys. 14, 1370 (2005)

24. Z. Jin, Q. Liu, Chinese Phys. 15, 1248 (2006)

25. M. Keeling, Proc. R. Soc. B 266, 859 (1999)

26. M.J. Keeling, M.E.J. Woolhouse, D.J. Shaw, L. Matthews, M. Chase-Topping, D.T. Haydon, S.J. Cornell, J. Kappey, J. Wilesmith, B.T. Grenfell, Science 294, 813 (2001) 